\section{Subcutaneous adipose tissue classification}

\author{
A. Sbarbati, ${ }^{1}$ D. Accorsi, ${ }^{2}$ D. Benati, \\ L. Marchetti, ${ }^{2}$ G. Orsini, ${ }^{3}$ G. Rigotti, ${ }^{4}$ \\ P. Panettiere
}

'Department of Neurological, Neuropsychological, Morphological and Motor Sciences, Human Anatomy and Histology Section, University of Verona ${ }^{2}$ Department of Specialised and Anaesthesial Surgery Sciences, University of Bologna

${ }^{3}$ Department of Clinical Sciences and

Stomatology, Marche Polytechnic

University, Ancona

${ }^{4}$ Second Division of Plastic and

Reconstructive Surgery, the Institute for Burns, and Regional Center for Breast Reconstruction, Ospedale Maggiore, Verona, Italy

\section{Abstract}

The developments in the technologies based on the use of autologous adipose tissue attracted attention to minor depots as possible sampling areas. Some of those depots have never been studied in detail. The present study was performed on subcutaneous adipose depots sampled in different areas with the aim of explaining their morphology, particularly as far as regards stem niches. The results demonstrated that three different types of white adipose tissue (WAT) can be differentiated on the basis of structural and ultrastructural features: deposit WAT (dWAT), structural WAT (sWAT) and fibrous WAT (fWAT). dWAT can be found essentially in large fatty depots in the abdominal area (periumbilical). In the dWAT, cells are tightly packed and linked by a weak net of isolated collagen fibers. Collagenic components are very poor, cells are large and few blood vessels are present. The deep portion appears more fibrous then the superficial one. The microcirculation is formed by thin walled capillaries with rare stem niches. Reinforcement pericyte elements are rarely evident. The sWAT is more stromal; it is located in some areas in the limbs and in the hips. The stroma is fairly well represented, with a good vascularity and adequate staminality. Cells are wrapped by a basket of collagen fibers. The fatty depots of the knees and of the trochanteric areas have quite loose meshes. The fWAT has a noteworthy fibrous component and can be found in areas where a severe mechanic stress occurs. Adipocytes have an individual thick fibrous shell. In conclusion, the present study demonstrates evident differences among subcutaneous WAT deposits, thus suggesting that in regenerative procedures based on autologous adipose tissues the sampling area should not be randomly chosen, but it should be oriented by evidence based evaluations. The structural peculiarities of the sWAT, and particularly of its microcirculation, suggest that it could represent a privileged source for regenerative procedures based on autologous adipose tissues.

\section{Introduction}

In obese individuals, subcutaneous fat can represent the most voluminous structure of the body and several data suggest that it plays a role in the pathogenesis of some severe diseases. ${ }^{1,2}$ Moreover, in regenerative medicine, methods based on autologous subcutaneous adipose tissues are getting more and more popular. ${ }^{3}$ In fact, among the various stem population identified by now, the mesenchymal adipocyte line proved to be the most promising in the perspective of clinical appliances, due to its abundance and availability. Subcutaneous adipose tissue represents an almost unlimited reservoir of stem cells that can be harvested with minimally invasive procedures. ${ }^{4}$ It is noteworthy that unlike all other body tissues, fat can significantly increase its mass with age. Moreover, an increasing number of experimental studies highlighted the huge neoangiogenic and immunomodulatory potentials of adipose stem cells, promoting their use in the therapy of ischemic and autoimmune diseases. ${ }^{5}$ Some animal model based studies and preliminary clinical trials demonstrated the therapeutic efficacy in neurologic and cardiovascular pathologies. Moreover, some clinical trials proved the lack of any of the complications pointed out by different stem cells regenerative therapy approaches. ${ }^{6}$ The currently more advanced clinical application is in plastic surgery where adipose cells revealed to be able to correct several pathologies and in particular scar lesions.

In such a context, the complex made of cell, extracellular and biochemical elements whom the adipose cell interacts with in its natural micro-environment (niche), proved to play a critical role. The implant of autologous stem cells enriched lipoaspirate rather than isolated stem cells could configure as the substitution of an atrophic niche with a physiologic one, where the stem niches can be forced to perform their normalizing role on the receiving tissue. The development of stem niches based therapies emphasizes the need for a precise characterization of the stromal components of subcutaneous adipose tissues, which is the
Correspondence: Prof. Andrea Sbarbati, Dipartimento di Scienze Neurologiche, Neuropsicologiche, Morfologiche e Motorie, Sezione di Anatomia e Istologia, Università di Verona, strada Le Grazie 8, 37134, Verona, Italy.

Tel. +39.045.8027155 - Fax: +39.045 .8027163$

E-mail: andrea.sbarbati@univr.it

Key words: adipocyte, adipose stem cells, fat transplantation, liposuction.

Received for publication: 19 August 2010.

Accepted for publication: 6 October 2010.

This work is licensed under a Creative Commons Attribution 3.0 License (by-nc 3.0).

(C) Copyright A. Sbarbati et al., 2010

Licensee PAGEPress, Italy

European Journal of Histochemistry 2010; 54:e48 doi:10.4081/ejh.2010.e48

most common source of stem niches.

Despite the metabolic and molecular behavior of these elements have already been characterized, ${ }^{8}$ a comparative analysis of the stromal components of adipose tissues (in particular of the collagenic scaffold wrapping the adipocytes in the different sites) has never been performed.

The lack of scientific evidence represents a serious knowledge gap so that the choice of a sampling site is based only on mere empirical or even random considerations.

Still, morphologic studies carried out until now generally gave great relevance to the abdominal fatty depots and only paid a scarce attention to the minor ones.

Information about such a structural organization cannot be obtained by means of biochemical techniques or optical microscopy, but requires ultrastructural evaluations. The features of such a scaffold could be the reason for the different resistance of adipocytes to the mechanical stimulations during harvesting, isolation and implantation. ${ }^{9}$

Moreover, the knowledge about the microcirculation and the stem cells niches associated to the different fatty depots is totally incomplete. Ultrastructural analysis techniques never used in the past are seemingly mandatory for the study of these parameters too.

In the present study we therefore analyzed a high number of subcutaneous adipose tissue samples harvested from the most common sources of adipose stem cells to determine their features and to highlight the tissue parameters that address them to a preferential use in the various conditions in reconstructive medicine. In particular, an integrated protocol of transmission and scan electron microscopy was used, providing an accurate tridimensional and sub-microscopic evaluation of these 
structures. The study of subcutaneous white adipose tissue by means of optical microscopy doesn't allow an adequate visualization of the structural tissue organization. In fact, the large dimensions of the cells, whose cytoplasm is occupied almost totally by a unique triglyceride drop, generates an apparently amorphous pattern inside which it is virtually impossible to understand the features of the organization. The intracellular space appears compressed among the triglyceride masses and its aspect appears not differentiable among the different tissue areas. Optical microscopy alone revealed scarcely useful also in the characterization of both the connectival stroma and the vasculo-stromal component of subcutaneous adipose tissue. A protocol based on comparative evaluations of scanning electron microscopy (SEM) and transmission electron microscopy (TEM) seemed to be more suitable for such aims. These techniques, in fact, can provide complementary information about the tridimensionality of collagenic and stem-vascular structures and about their fine composition.

\section{Materials and Methods}

Forty female patients with age ranging from 30 to 67 years (average: 51.3 ys) underwent fat harvesting from September to October 2009 for autologous fat implant. A mixture of $0.5 \%$ xylocaine + epinephrine 1:200,000 in saline solution was injected before suction. Fat harvesting was performed by a $3 \mathrm{~mm}$ cannula (1-hole, bullet tip) connected to a 10 cc syringe for vacuum; fat was then gently washed in saline and decanted. Centrifugation was not performed. Harvesting was performed in 10 different sites: arms, armpits, pectoral, periprosthetic tissue (breast prostheses, irradiated or non-irradiated), abdomen, pubic, hips, trochanters, inner face of the knees and heels.

For ultrastructural examination, we used methods applied in previous studies on white adipose tissue (WAT): specimens withdrawn in these works were also used as controls. ${ }^{1}$

For TEM, parts of the specimens were immediately fixed in $2.5 \%$ glutaraldehyde in Sorensen buffer for $2 \mathrm{~h}$, postfixed in 1\% osmium tetroxide in Sorensen buffer for $1 \mathrm{~h}$ dehydrated in graded acetones, embedded in EponAraldite and cut with an Ultracut E Ultra microtome (Reichert, Wien, Austria). Ultrathin sections were stained with lead citrate and observed in an EM10 electron microscope (Zeiss, Oberkocken, Germany). The semithin sections were stained with Toluidine blue.

For SEM the specimens were fixed in 2.5\% glutaraldehyde in Sorensen buffer for $2 \mathrm{~h}$, post- fixed in $1 \%$ osmium tetroxide in Sorensen buffer for $1 \mathrm{~h}$ and dehydrated in graded acetones (Fluka, St. Louis, M0, USA). The specimens were then treated by critical point dryer (CPD 030; Balzers, Liechtenstein) and coated by gold, mounted on stubs and observed in an XL 30 ESEM (FEI- Philips). Data about adipocyte size were obtained by SEM examination.

\section{Results}

The combined analysis by transmission and scan electron microscopy allowed us to define the three main different patterns in the subcutaneous WAT described below.

Fatty depots appear to be differentiable into distinct adipose tissue typologies. However, it should be clarified that also in the same depot there can be evident differences that can be correlated to the superficial or deep localization or to the patient's age. In general, the depots subject to mechanic stresses present a larger connective component. There are not only qualitative differences, but also quantitative ones and intermediate states among the different typologies can be evidenced.

It should also be clarified that, inside larger depots, the aspect could be inhomogeneous. In the abdomen and in the hips, for example, the deep layer appeared richer in fibrous structures. Moreover, in older patients there is a larger amount of collagen than in younger ones. Such an increase appears to be both an increase in the more coarse connectival mesh and a thickening of the peri-adipocyte meshes. The average diameter tends to increase with age, while the number of peri-vascular stem cells tends to reduce. In the older patients a moderate thickening of vascular walls was also observed, with duplicated basal membranes and homogeneous thickenings at times.

\section{Classification of subcutaneous depots}

Subcutaneous depots only apparently present structural and ultrastructural homogeneity. Actually, they form a very polymorphous and variegate sub-family of tissues due to the different roles played by each single district.

In general, three kinds of adipose tissues can be distinguished, based on their structural and ultrastructural features. Such types differ by both the adipocyte and the vasculo-stromal components. Ranging from type one to three, a progressive increase in stroma (with different features) can be observed. However, it should be underlined that in different areas, the individual depots can be made by mixtures of different types of adipose tissues.

\section{Type \#1 deposit white adipose tissue}

The type \#1 adipose tissue (Figure1 A,E) is located essentially in large depots in the abdominal area (peri-umbilical). It is a typical non-lobulated adipose tissue that could be defined as metabolic fat due to its large lipidic mass and its very poor collagenic component (non-stromal). It is characterized by large adipose cells: the average diameter, in our experimental conditions, was $95 \mu \mathrm{m}$. It presents few capillaries in comparison to other WATs. The TEM demonstrates that cells are closely packed while at SEM a weak reticulum of isolated collagen fibers is visible. In particular, at SEM the fibrillar network connecting the contiguous adipocytes is extremely weak or virtually absent. These cells appear not to be wrapped by a real collagen fibers basket, which can instead be found in other types of WAT. The adipose cells form a sort of a syncytium where the extracellular space is extremely thin. The microcirculation is formed by thin walled capillaries with rare stem niches. Pericyte reinforcement elements are rarely evident. These adipocytes, not being separated by collagen, tend to adhere each other with parallel membrane plates. In the older patients the mesh was thicker, taking a coarse aspect with large shoots forming a loose meshed reticulum. Peri-vascular macrophages are not infrequent.

\section{Type \#2 structural white adipose tissue}

The type \#2 adipose tissue (Figure $2 \mathrm{~A}, \mathrm{D}$ ) is more polymorphous and variable from site to site, as it plays different roles based on the relationships with the surrounding structures. Such variability can also be appreciated as differences in tissue consistency during harvesting. In general, it is a more stromal fat and it is located in much limited adipose areas, usually rich of muscular tissue.

The type \#2 fat is characteristic of more localized depots, where its function goes undoubtedly beyond the depot itself: in such areas, the adipose tissue appears mainly finalized to mould, that is to give a shape to the structure. The fat in trochanters, sovrapubic area, arm pits, inner faces of the knees, thighs, arms, pectoral and mammary areas and hips all pertain to this group. The average diameter of adipocytes is smaller than in dWAT: about 81 $\mu \mathrm{m}$. Greater diameters can be found in the inner faces of the knees $(92 \mu \mathrm{m})$ and in the trochanters $(86 \mu \mathrm{m})$.

It is a non-lobular adipose tissue with a variable collagenic component, often forming coarse shoots. They appear relatively easy to dissociate due to their structure. At times the stroma appears fairly good and well vascularized. Staminality is generally fair. In fact, some elements related to the basal membrane (poor- 
ly differentiated or in course of differentiation) can be found in the peri-capillary spaces. Such elements are generally rich of polyribosomes and have few other organules made of isolated endoplasmic reticulum cisternae. Moreover, capillaries can show basal membrane duplications or thickenings, mostly in aged people. Rare macrophagic elements are generally present. Among the various depots, some peculiar aspects can be noticed in those undergoing specific mechanic stimulations in the lower limbs. For example, the depots on the inner faces of the knees and in the trochanteric areas present a quite thin connectival mesh, almost mesenchymal-like. In scan images, the peri-adipocyte baskets are characterized by large meshes made of isolated collagen fibers. Such basket often tends to detach from the plasmalemma of the adipocytes (that in these areas appears uniformly smooth).

\section{Type \#3 fibrous white adipose tissue}

The type \#3 adipose tissue (Figure 3 A,C) has a remarkable fibrous component and is characterized by a well defined mechanic function. It can generally be found in areas where the mechanic stress can be considerable. Adipocytes are therefore smaller, with a thick fibrous shell wrapping them one by one. At SEM, a tight reticulum of thickened collagen fibers is visible in a thick layer around the adipocytes. Such a reticulum can be visible also at optical microscopy, but it appears clearly only at TEM. This kind of adipose tissue, characterized by a conspicuous prevalence of collagenic stroma can present in lobular or non-lobular subtypes.

\section{Lobular subtype}

It can be found for example in the calcaneal fat pad; it is a lobular adipose tissue with micro and macro chambers delimited by connective septa. The tissue is well vascularized, with capillaries characterized by a thick endothelium, duplicated basal membranes and covered by velamentous cells. The adipocytes are large (97 $\mu \mathrm{m})$ and covered by thick connective sheaths made of very thick collagen bundles. At SEM, the sheaths appear as compact, high density layers that prevent the single meshes to be caught a glimpse of due to the tight apposition of collagen fibers. However, the outer surface of the basket appears almost regular and easy to dissociate from the stromal collagen. Therefore, the sheaths appear as structures with an evident morphologic identity, closely associated to the plasmalemma of the adipocyte from which they seem to be separated by no visible space at TEM.

\section{Non-lobular subtype}

It is a tissue with a maximum degree of fibrosis. It can be found, for example, in the periprosthetic capsules (both irradiated and non irradiated). It is a hard adipose non-lobulated tissue, with a strong stromal component. Vascularization is florid and macrophages and at times elongated elements with a poor lipidic content (that can be referred to adipocyte line in course of differentiation), can be found around microvessels.

\section{Discussion}

In the present work, the comparative systematic analysis of human subcutaneous tissues performed with ultrastructural methods also in areas never studied before led to the description of a structure never described before: the collagenic peri-adipocyte basket. Such a structure probably plays an important role in the preservation of cell integrity and it can consequently influence the results in fat harvesting procedures and in autologous fat transplant. Based on the morphology of the basket, three different types of subcutaneous WATs can be patently identified and it is not surprising that the areas more subject to mechanic stress present more developed collagenic peri-adipocyte baskets.

An important data emerging from the present study is therefore the polymorphism of the subcutaneous adipose tissue. In fact, despite the common idea that fat is contained in an almost homogeneous cavity, the comparative systematic analysis of human samples demonstrated that the adipose organ appears as a very differentiated entity. This leads to realize that it should be more appropriated to use the terms subcutaneous adipose tissues instead of subcutaneous adipose tissue. The data obtained by groups of patients of different ages suggest that such a specialization could be in part related to the length of human life as it is less evident in the common laboratory species, whose average life span is generally no longer than 24 months. It appeared to be very difficult to appreciate these differences at mere optical microscopy and this is probably the main reason why they have never been underlined in preceding studies led with different aims. The differences found seem to be mainly related to
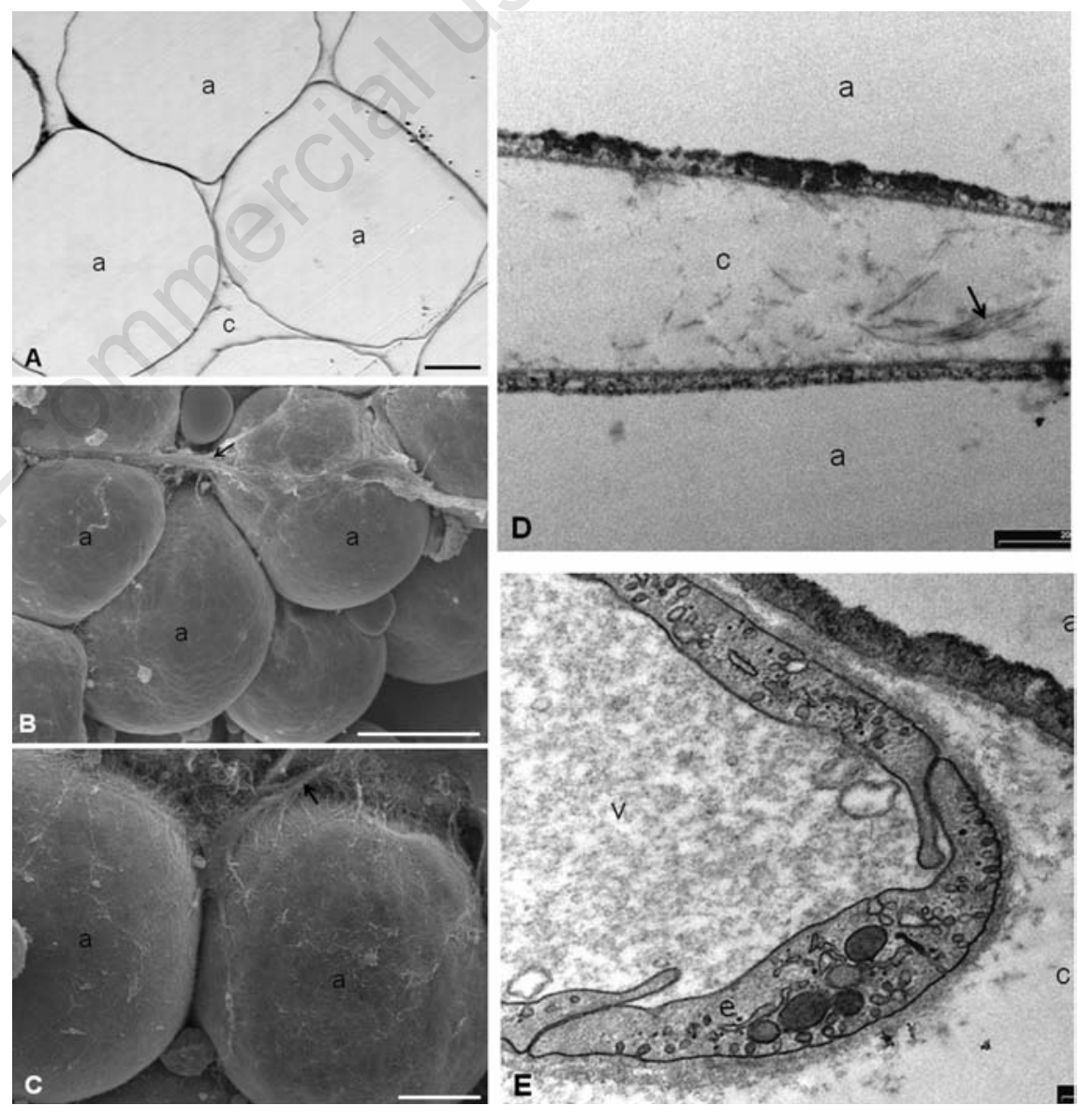

Figure 1. Abdominal adipose tissue: the tissue is characterized by large adipose cells and a poor collagenic component. (A) Light microscopy (scale bar: $15 \mu \mathrm{m}$ ). (B)SEM (scale bar: $50 \mu \mathrm{m}$ ). (C) SEM (scale bar: $20 \mu \mathrm{m}$ ). The adipocytes are surrounded by a thin capsule of collagen fibres. Panel D, TEM (scale bar: $2000 \mu \mathrm{m})$. Panel E, TEM (scale bar: $500 \mu \mathrm{m})$. TEM images confirm that only sparse collagen fibres surround the adipocytes. 
the composition of the stromal compartment and it patently emerged that the differences can be substantially connected to the functional specializations of the depots in the various sites. These results about regional differences in morphology confirm the data indicating that there are regional differences in the metabo-
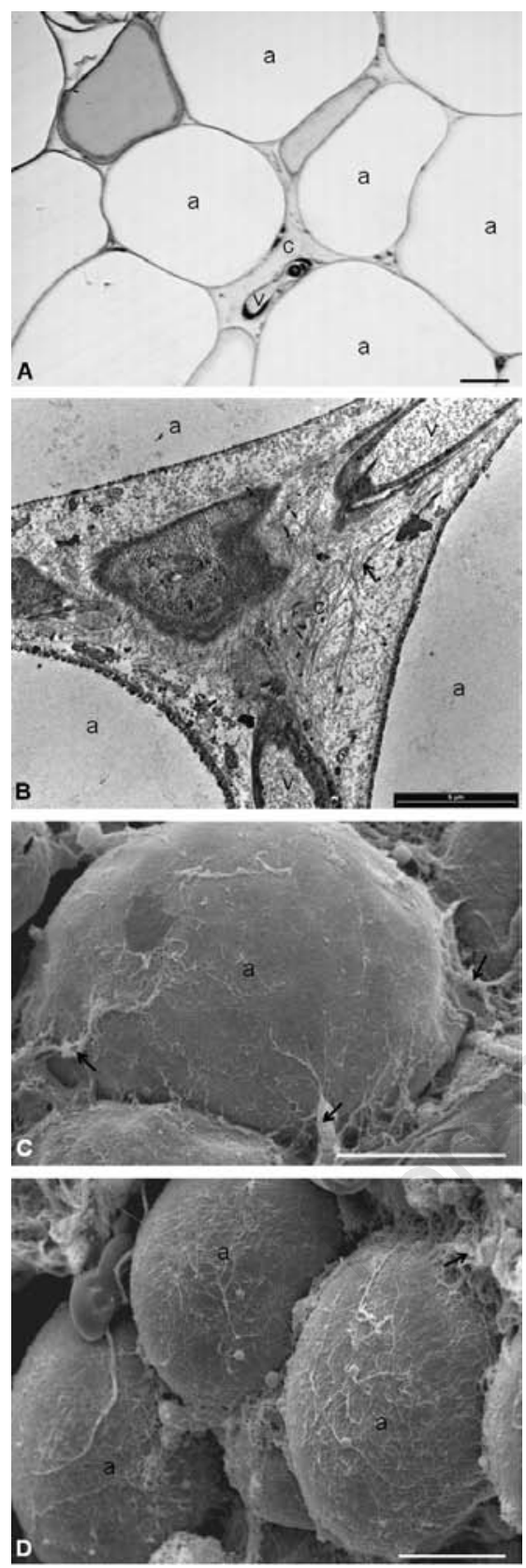

Figure 2. Structural adipose tissue. The type 2 adipose tissue is characterized by a variable collagenic component. (A) light microscopy (scale bar: $15 \mu \mathrm{m}$ ). (B) TEM (scale bar: $5 \mu \mathrm{m}$ ). The picture shows a stroma rich in collagen fibers and well vascularized. (C) SEM (scale bar: $35 \mu \mathrm{m})$. In this area the connective capsule is thin and partially detached. (D) SEM (scale bar: $20 \mu \mathrm{m}$ ). The picture shows adipocytes covered by a relatively dense connective capsule. lism of subcutaneous fatty depots. ${ }^{10,11}$ Lipids are mobilized at a slower rate but synthesized at a higher rate in the femoral than the abdominal region. Fasting is accompanied by an increased rate of fat mobilization and a decreased rate of fat synthesis in all fatty depots. These changes are, however, more pronounced in abdominal than in femoral fat. There are also regional differences in the hormonal regulation of fat metabolism in obesity. The action of insulin is most pronounced in the femoral region whereas the one of catecholamines is most marked in the abdominal area. The regional differences in hormone action are further enhanced during therapeutic fasting. ${ }^{10}$ Abdominal adipocytes from
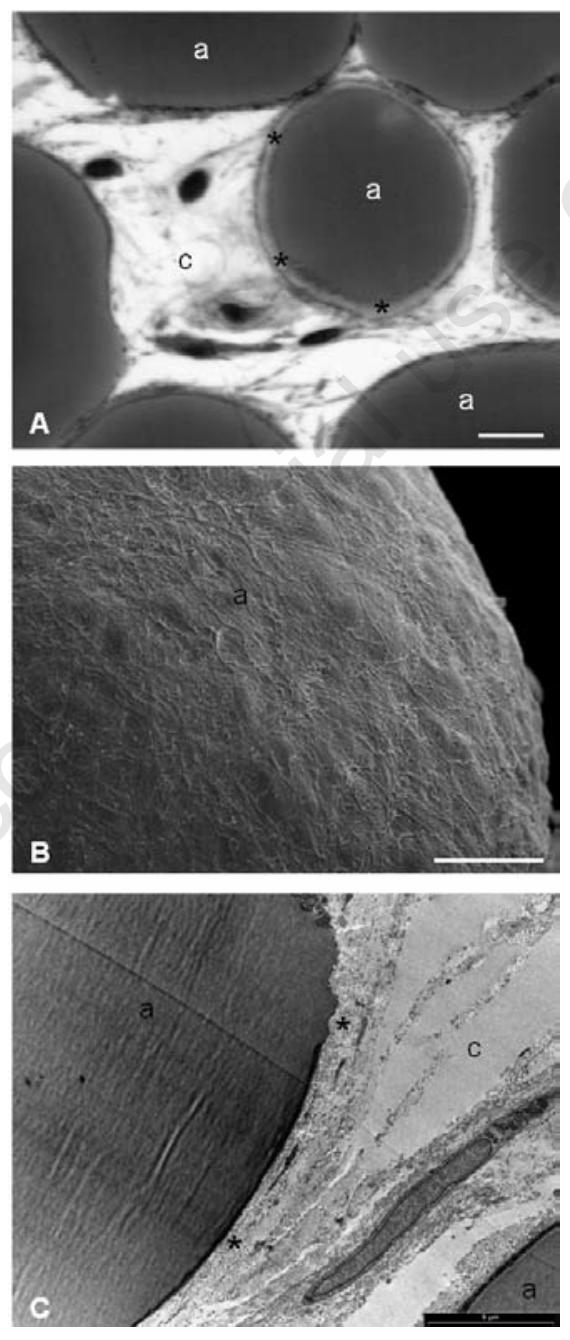

Figure 3. Fibrous adipose tissue with a remarkable fibrous component. (A) Light microscopy (scale bar: $15 \mu \mathrm{m}$ ). (B) SEM (scale bar: $10 \mu \mathrm{m})$. A tight reticulum of thickened collagen fibers is visible in a thick layer around the adipocytes. (C) TEM (scale bar: 5 $\mu \mathrm{m})$. The adipocytes are covered by thick connective sheaths made of very thick collagen bundles. a, adipocyte; $c$, connective tissue; $v$ blood vessel; e, endothelium; $\uparrow$ collagen bundle; * pericellular connective capsule. females showed a 40 times lower alpha 2adrenergic antilipolytic sensitivity than did gluteal adipocytes. ${ }^{11}$

While previous studies mainly focused on the adipocyte compartment, the present study aimed at a detailed analysis of the stromal compartment. Based on TEM and SEM analysis, we could define three main types of subcutaneous fat that can be named as dWAT, sWAT and fWAT.

The main difference among the three types is definitely the morphology of a structure that was never clearly identified before: the periadipocyte collagenic basket. Such a structure appears seemingly important in determining the mechanic features of the tissue and presents patent differences in the three types. In the dWAT, the basket is incomplete and extremely weak so the membranes of the adipocytes are tightly adherent. Usually, only isolated collagen fibers settle in the virtual space. As whole, the tissue appears as a sort of syncytium made of large dimension tightly adherent elements. So, such an aspect appears characterized by a huge lipidic collection with a poor stromal component. The difficulty of dissociation of the adipocyte depots during harvesting could be related to the tight adherence among the cells. This could also imply a higher cellular damage during harvesting because traction and suction could break the cells instead of separating them.

Further studies are necessary to confirm this hypothesis. The features of the microcirculation indicate that the dWAT is a slow turnover tissue with a low staminality, even if data from other techniques are mandatory to confirm morphologic findings.

In the sWAT, the collagenic peri-adipocyte basket is well structured and associated to an on average more cellular microvascular compartment compared to what was observed in the large abdominal depots. This could be related to the mechanic stress that the depots or part of them are subject to. In fact, sWAT is typically located in areas that underwent to a more pronounced exposition to traumatism with respect to dWAT. It should be pointed out that the sWAT takes different morphologies depending on the depots and on the age of the patient.

In the fWAT, the collagenic peri-adipocyte basket appears as enormously thick and made of collagen parallel bands. Such feature is added to an absolutely peculiar microcirculation morphology, characterized by thick walled vessels with, at times, duplicated basal membranes. These features are probably related to the peculiar tissue mechanic performances that can be found in pathologic or, more rarely, physiologic situations such as in the calcaneal pad. In this case, it is evident that the functional performances under load of "soft" elements 
like adipocytes would be unbelievable without a noteworthy collagenic apparatus. It is significant the fact that despite the absolutely peculiar features of this tissue, it has never been ultrastructurally studied in detail. The use of such methodologies clearly shows that the main feature of fWAT is not only a greater amount of collagen, but rather mainly its different set-up that takes the shape of collagenic peri-adipocyte membranes. The microcirculatory morphology is also absolutely peculiar due to the presence of some ultrastructural aspects like the endothelial thickness, the presence of perivascular elements, but mostly the duplication of basal membranes, suggesting a rapid cell turnover, probably in response to chronic traumatism.

In conclusion, the present study describes a new morphologic structure: the peri-adipocyte basket. It demonstrates the existence of different varieties of WAT and that noteworthy differences are present in the connectival and micro-vascular compartments. Moreover, this work provides practical indications for optimization of harvesting procedures in the treatments based on autologous WAT.

Our data suggest that the best results in harvesting procedures and in subsequent implant can probably be obtained by the sWAT. In this tissue the elements are separated by a thin collagen voile and the microcirculation is rich of stem elements. Such features could be related to the chronic stimulation status to which the depots undergo leading to a cytoplasmatic degree of activation of the pericyte elements. This makes sWAT the ideal tissue for staminality restoring therapies based on the creation of ectopic stem niches. ${ }^{12}$

In particular, our data suggest as particularly noteworthy those sWAT depots where the collagenic mesh is particularly weak, such as the trochanteric ones or those in the inner face of the knees. Moreover, data from SEM seem to demonstrate that in such depots the collagenic sheaths can easily be detached from the adipocytes. This feature could be a further factor explaining the optimal consistency of the tissue. The weakness of the connectival baskets and the easy detachability of adipocytes seem to favor tissue harvesting. In our experience in these depots the dissociability of the adipose elements is higher.

Finally, these variants of sWAT have a strong vasculo-stromal fraction, very rich in already activated stem cells. Such features are probably determinant factors in the process of reconstructing ectopic stem niches in the damaged tissues. Therefore, this work suggests that, in regenerative procedures based on transplant of autologous adipose tissues, the site of harvesting should not be randomly chosen, but it should be indicated by evidence based evaluations. The structural peculiarity of sWAT, and in particular of its microcirculation, suggests that this tissue could represent a privileged source for harvesting in regenerative procedures based on autologous adipose tissues..$^{13,14}$

\section{References}

1. Sbarbati A, Osculati F, Silvagni D, Benati $\mathrm{D}$, Galiè M, Camoglio FS, et al. Obesity and inflammation: evidence for an elementary lesion. Pediatrics 2006;117:220-3.

2. Cinti S. The adipose organ. 1999 Ed. Kurtis, Milan, Italy.

3. Rigotti G, Marchi A, Sbarbati A. Adiposederived mesenchymal stem cells: past, present, and future. Aesthetic Plast Surg 2009;33:271-3.

4. Krampera M, Marconi S, Pasini A, Galiè M, Rigotti G, Mosna F, et al. Induction of neural-like differentiation in human mesenchymal stem cells derived from bone marrow, fat, spleen and thymus. Bone 2007;40:382-90.

5. Constantin G, Marconi S, Rossi B, Angiari S, Calderan L, Anghileri E, et al. Adiposederived mesenchymal stem cells ameliorate chronic experimental autoimmune encephalomyelitis. Stem Cells 2009;27: 2624-35.

6. Rigotti G, Marchi A, Stringhini P, Baroni G, Galiè M, Molino AM, et al. Determining the Oncological Risk of Autologous Lipoaspirate Grafting for Post-Mastectomy Breast Reconstruction. Aesthetic Plast Surg 2010;34:475-80.

7. Rigotti G, Marchi A, Galiè M, Baroni G, Benati D, Krampera M, et al A. Clinical treatment of radiotherapy tissue damage by lipoaspirate transplant: a healing process mediated by adipose-derived adult stem cells. Plast Reconstr Surg 2007;119:1409-22.

8. Peroni D, Scambi I, Pasini A, Lisi V, Bifari F, Krampera M, et al. Stem molecular signature of adipose-derived stromal cells. Exp Cell Res 2008;314:603-15.

9. Galiè M, Pignatti M, Scambi I, Sbarbati A, Rigotti G. Comparison of different centrifugation protocols for the best yield of adipose-derived stromal cells from lipoaspirates. Plast Reconstr Surg 2008; 122:e233-4.

10. Arner P. Site differences in human subcutaneous adipose tissue metabolism in obesity. Aesthetic Plast Surg 1984;8:13-7.

11. Wahrenberg $H$, Lönnqvist $F$, Arner $P$. Mechanisms underlying regional differences in lipolysis in human adipose tissue. J Clin Invest 1989;84:458-67.

12. Sbarbati A, Galiè $M$. The niche theory for fat graft survival. Pages 15-30 in: Fat injection. From filling to regeneration. Coleman SR and Mazzola RF, eds. 2009, Quality Medical Publ., Inc, St. Louis, MI, USA.

13. Panettiere P, Marchetti L, Accorsi D, Del Gaudio GA. Aesthetic breast reconstruction. Aesthetic Plast Surg 2002; 26:429-35.

14. Panettiere P, Marchetti L, Accorsi D. The serial free fat transfer in irradiated prosthetic breast reconstructions. Aesthetic Plast Surg 2009;33:695-700. 